\title{
Indagini tra le carte di Giovanni Orelli. Sulla genesi del romanzo Il sogno di Walacek
}

\author{
Giovanna CoRDiBELla \\ Universität Bern
}

\begin{abstract}
Il saggio si propone di illustrare alcuni risultati emersi dallo studio delle carte del fondo di Giovanni Orelli presso l'Archivio svizzero di letteratura (ASL). Dopo un'introduzione dedicata alla storia del fondo, vengono sottoposti ad analisi alcuni materiali avantestuali del quarto romanzo di Orelli, Il sogno di Walacek, al fine di dare rilievo al profilarsi - fin dai più antichi appunti e abbozzi - di un aspetto peculiare dell'opera: l'istituzione nella prosa orelliana di frequenti riferimenti intermediali, ben documentati anche dalle carte d'archivio, nelle quali numerosi sono i rimandi al quadro Alphabet I di Paul Klee.
\end{abstract}

Keywords: archivio, Il sogno di Walacek, intermedialità, Paul Klee.

Perché, pensai, non buttar giù [da un ponte] le mie cartacce, gli scartafacci, come li chiama snobbandoli Benedetto Croce? Mi dissi di no. Non avrei affidato i miei tentativi di scrittura, i manoscritti a quell'inesorabile correttore che è il fuoco, o a un'acqua che tutto porta via. No, li avrei affidati al museo, che per le carte è l'archivio.

Giovanni Orelli, «Perché andare a Berna...» (20II)

\section{Gli scartafacci nella «fossa degli orsi»: il fondo di Orelli all'ASL}

Primo autore italofono a lasciare nel 1993 le proprie carte all'Archivio svizzero di letteratura (ASL), Giovanni Orelli ha notoriamente inaugurato la collezione di fondi in lingua italiana, oggi giunta ormai a una consistenza notevole, di questa prestigiosa istituzione archivistica svizzera. La scelta di destinare i propri «scartafacci» alla «fossa degli orsi» (Orelli I993: 40), per riprendere un'icastica e autoironica definizione orelliana, trova come noto la propria spinta propulsiva in più ragioni. Si è trattato in prima linea, come l'ha definita Orelli stesso, di una ben meditata «scelta [...] di natura simbolica e di carattere politico", dettata da una ben salda convinzione autoriale: la necessità di rafforzare «le corrispondenze biunivoche tra le regioni linguistiche della Svizzera», senza «lasciarsi tentare dalle sirene dell'autarchia» (4I). Da qui l'identificazione dell'ASL nella capitale elvetica come sede eletta alla custodia delle proprie carte a scapito di sedi italiane (come il Centro Manoscritti di Pavia) o svizzero-italiane (come la Biblioteca cantonale 
di Lugano), con predilezione appunto, in antitesi al frammentismo e alle tentazioni autarchiche, per l'istituzione nazionale deputata centralmente a questo ruolo. Le dichiarazioni d'autore intorno a questa risoluzione lasciano inoltre emergere un più generale atteggiamento di Orelli, qui altrettanto degno di nota, nei confronti dei propri «scartafacci». È l'atteggiamento di un autore formatosi in un secolo, il Novecento, che può ormai considerare del tutto conclusa l'affermazione di un decisivo «Paradigmenwechsel» (Bülow von 20I6: II4) nella prassi di gestione autoriale delle proprie carte. Per autrici e autori di quest'epoca è infatti ormai ampiamente diffusa la consuetudine, dopo questo cambio di paradigma, di conservare con scrupolo i propri manoscritti, dattiloscritti, bozze di stampa, così come gli schizzi, le note progettuali e i compositi dossier relativi alle proprie opere, nella piena consapevolezza di come tali materiali costituiscano non secondarie testimonianze della genesi e della tradizione di queste ultime. Orelli condivide in pieno tale prassi conservativa. Non solo s'impegna a evitare la dispersione delle proprie carte e ad assicurarne la loro salvaguardia, ma si preoccupa inoltre di garantirne anche la persistenza e la conservazione future, optando appunto di «affidarle al museo, che per le carte è l'archivio» (Orelli 2oııa: 82).

Chi si avventuri a perlustrare le stanze di questo affascinante luogo della memoria culturale che è l'archivio, addentrandosi nello specifico in un esame dei materiali del fondo orelliano presso l'ASL, è anzitutto confrontato con la considerevole consistenza del fondo (incrementato anche in tempi recenti, dopo la scomparsa dell'autore, con il trasferimento a Berna nel 2018 delle sue carte più tarde) e inoltre con la consultazione di materiali documentari la cui natura è altamente composita. All'ampia mole del lascito non hanno finora corrisposto ricerche altrettanto estese tra i suoi materiali. Ancora parzialmente in fase di catalogazione, le carte di Orelli sono state infatti finora oggetto soltanto di alcune indagini circoscritte che si sono di volta in volta focalizzate sull'esame della genesi e dello sviluppo variantistico di alcune opere (Puddu 2013a, 2013b; Ganzoni 2013: 92-93), sulla loro storia editoriale (Soldini 2018) o sui rapporti (epistolari e non solo) intrattenuti da Orelli con esponenti della scena poetica contemporanea (Pedroni 20I8). Il presente saggio intende contribuire alla direzione di ricerca avviata da questi contributi e presenta alcuni dei risultati emersi da analisi di materiali relativi al quarto romanzo di Orelli: Il sogno di Walacek (I99I). Il dossier archivistico su quest'opera ha una considerevole consistenza e comprende, accanto ad appunti, abbozzi, alla trafila delle stesure, anche diversi materiali documentari che sono diretta testimonianza delle ricerche che Orelli ha svolto in fase di progettazione e di elaborazione al romanzo ${ }^{1}$. La prospettiva

I Un inventario del dossier archivistico relativo al Walacek è consultabile al seguente indirizzo: http://ead.nb.admin.ch/html/orelli_A.html\#A-I-i (consultato il I7 marzo 20I9). Tra i materiali documentari raccolti da Orelli nel corso delle sue ricerche si segnalano: una corrispon- 
d'analisi qui adottata implicherà soltanto lo studio selettivo di alcune carte di questo ricco e articolato dossier. Al centro dell'indagine vi sarà l'esame di un particolare aspetto della genesi de Il sogno di Walacek che, come vedremo, risulta anche centrale per lo studio della sua tecnica diegetica e, più in generale, del principio costruttivo del romanzo.

\section{In principio un quadro: sulla genesi de Il sogno di Walacek}

Orelli ha fornito in più occasioni dettagli intorno alla prima fase del lavoro compositivo al Walacek, illustrando inoltre la situazione extra-testuale che ha condotto al delinearsi dell'idea originaria dell'opera. Su quest'ultimo punto egli offre anche ragguagli in una delle sue più tarde interviste:

Il sogno di Walacek è nato e si è sviluppato per continue associazioni di idee [...]. Una mattina, prima di partecipare a un'assemblea della commissione dell'Unesco in programma a Berna, andai a visitare il museo (allora provvisorio) di Paul Klee vicino alla stazione. In un angolo era eccezionalmente esposto [...] Alphabet I, un quadro minore di Klee [...]. Quando lo vidi pensai subito al fatto che Klee si era comportato come una perfetta massaia che, quando deve sbucciare le patate, non sceglie la pagina dei necrologi, perché le sembrerebbe di compiere un atto irriverente [...]. Ma sceglie la pagina dello sport, perché con lo sport - nonostante sia la religione del nostro tempo - tutto sommato non si offende nessuno. E quindi Klee, credo volutamente, aveva anche lui scelto lo sport. E guardando bene la pagina, sotto il pennello dell'artista, si potevano vedere le squadre e i nomi dei calciatori. Lì, in quel momento, è nata l'idea del Sogno di Walacek [...] (Orelli 20II: 4-5).

Sulla base di questa testimonianza autoriale l'idea originaria del romanzo deve quindi essere fatta risalire a una visita di Orelli a una mostra di Klee al Kunstmuseum di Berna e, in particolare, alla contemplazione del quadro Alphabet I (fig. I), una cui riproduzione fotografica verrà anche edita nella princeps de Il sogno di Walacek e collocata nella vera e propria «soglia» del testo, direttamente dopo il frontespizio (Orelli I99ia: [2]).

L'attenzione dell'autore cade soprattutto su alcuni elementi del quadro, in prima linea sull'anomalo supporto su cui Klee dipinge, vale a dire la pagina sportiva dell'edizione del 19 aprile 1938 della National-Zeitung di Basilea dove è riportata la cronaca di una partita di calcio, la finale della coppa svizzera tra i Grasshoppers di Zurigo e il Servette di Ginevra, che si è tenuta

denza con il FC Servette e con Génia Walacek (che comprende tra l'altro anche un Proust Questionnaire, adattato da Orelli e sottoposto al calciatore per avere informazioni sulla sua personalità), una collezione di articoli di giornale sulla storia calcistica, così come documenti relativi alla morte del pittore Paul Klee e alla sua cremazione. 


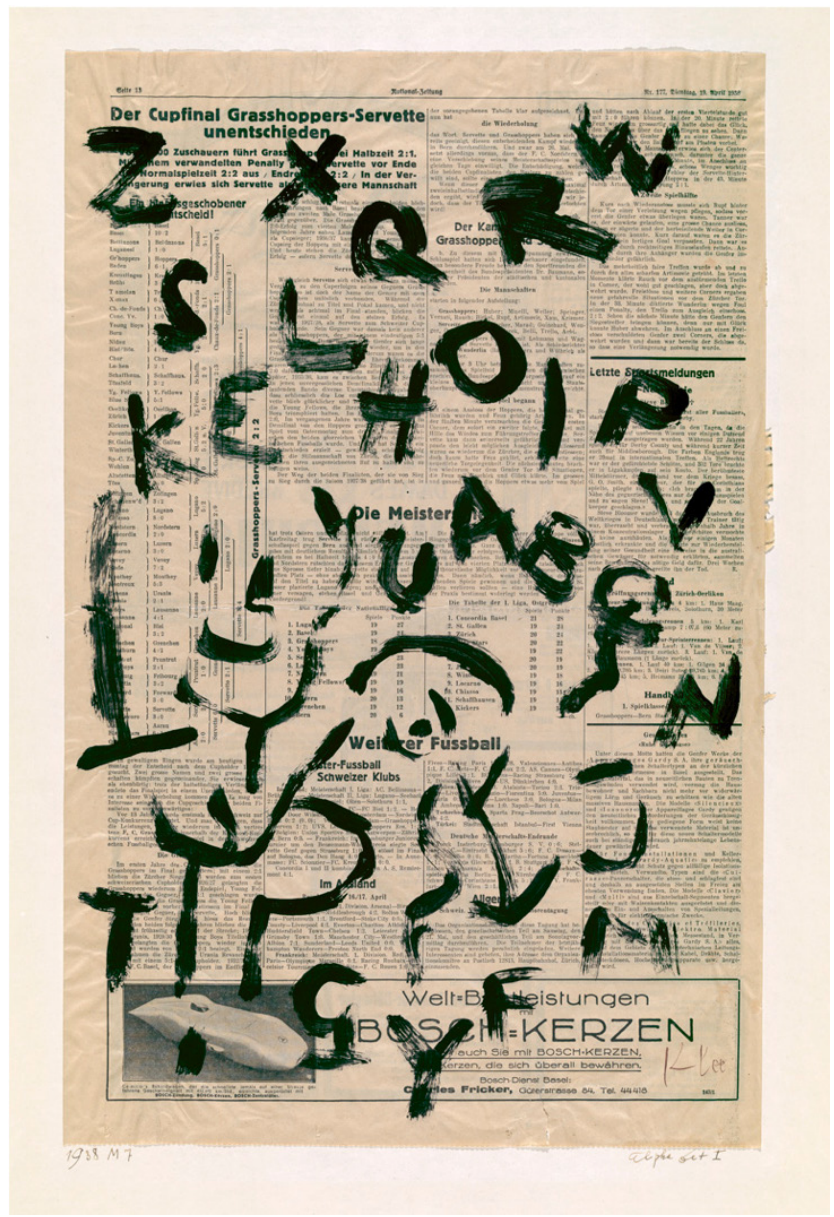

Fig. I: Paul Klee, Alphabet I, 1938. (c) Zentrum Paul Klee, Bern.

allo stadio Wankdorf di Berna il giorno prima. Nel fondo di Orelli è conservato un documento (fig. 2 ) che consente una ricostruzione più accurata di questo originario confronto di Orelli con Alphabet I e che testimonia quali primi appunti siano stati effettivamente presi di getto dall'autore durante (o subito dopo) la visita al Kunstmuseum. Orelli ha annotato le sintetiche notizie sul primo foglio a sua disposizione, il verso di una ricevuta di un hotel zurighese emessa il 2I febbraio $1989^{2}$, data che può essere dunque senz'altro

2 La carta con questi primi appunti (ASL, A-I-i/2-I) può essere quindi considerata come il documento cronologicamente più antico dell'intero dossier archivistico relativo al Walacek. 
assunta come termine post quem a cui far risalire l'avvio della composizione del romanzo.

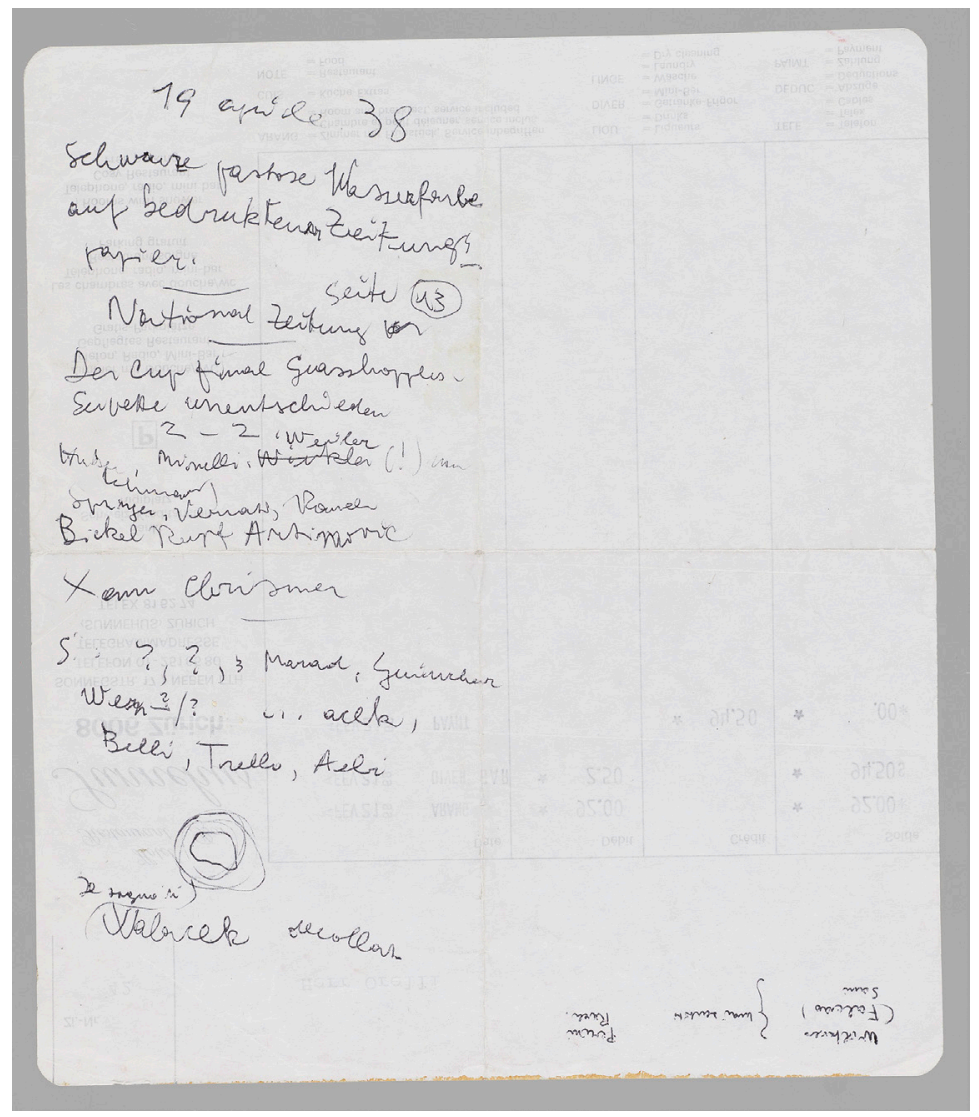

Fig. 2: Giovanni Orelli, Appunti relativi ad «Alphabet I» (ASL, A-I-i/2-I).

Questo foglio di appunti merita un'analisi accurata. Orelli vi ha in primo luogo annotato una data («I9 aprile 38») che corrisponde a quella del giornale su cui Klee ha dipinto, con a seguire una sintetica descrizione in tedesco di Alphabet I: «Schwarze pastose Wasserfarbe auf bedruckt[em] Zeitungspapier» («Inchiostro nero pastoso su carta stampata di giornale»), probabil-

3 In queste annotazioni Orelli denota un'incertezza nella trascrizione di un luogo della descrizione di Alphabet I in tedesco («auf bedrucktenen Zeitungspapier» - se ben si interpreta la correzione orelliana - al posto della forma corretta «auf bedrucktem Zeitungspapier»). Il refuso si trova riprodotto anche nella princeps del romanzo (Orelli I991: I4), a conferma del rapporto genetico tra gli appunti e questo luogo dell'opera, e verrà riproposto anche nella sua 
mente un appunto dalla didascalia del quadro offerta dal museo. Questa descrizione, in tedesco con corredo della sua traduzione italiana, verrà ripresa testualmente nelle prime pagine del romanzo (Orelli i99Ia: I4), laddove il narratore racconta come sia nato Alphabet I (come Klee abbia dunque dipinto questo quadro a Berna nel I938, strappando, forse non del tutto a caso, questa pagina dalla National-Zeitung). Orelli appunta anche il numero della pagina di questo giornale scelta da Klee («Seite I3»), un numero su cui nel romanzo verranno fatte diverse speculazioni (I7-I8). Dopo la trascrizione del titolo dell'articolo che incuriosisce Orelli e che catalizza la sua attenzione, segue nella parte inferiore della carta la parziale annotazione delle formazioni delle due squadre che si sono fronteggiate nella finale. L'occhio di Orelli cade in questo caso su un dettaglio dell'opera di Klee: la lettera «O» che il pittore ha tracciato con inchiostro nero e che in parte nasconde alcuni nomi di giocatori, tra questi appunto Walacek, una mezzala del Servette di Ginevra. Orelli annota in questo caso dei puntini di sospensione e soltanto alcune lettere («acek»), leggibili nel quadro, del nome della mezzala. Segue infine una riproduzione iconica della lettera «O» di Klee e una prima nota che include lo stesso titolo del romanzo: «Il sogno di Walacek» (con a latere l'enigmatico: «decollar»).

Non v'è alcun dubbio che questo documento d'archivio costituisca una delle testimonianze più antiche relative alla genesi del romanzo, nel quale ripetuti ed espliciti saranno infatti i riferimenti a Alphabet I di Klee e dove tra i principali personaggi, accanto al pittore originario di Berna, proprio il giocatore Génia Walacek si profilerà come protagonista. Dopo la visita al Kunstmuseum Orelli inizia in effetti a progettare un'opera narrativa - in un primo tempo un «lungo racconto» (Orelli I989: 39I), successivamente un vero e proprio romanzo - che ruota intorno alle vicende del pittore «degenere» Klee e del rifugiato Walacek, due apolidi e figli, a tutti gli effetti, del xx secolo. Orelli darà appositamente avvio a vere e proprie ricerche sul giocatore e sugli ultimi anni di Klee, come documentano le carte d'archivio. Ma l'universo narrativo che si delinea nel romanzo è ben più ampio e complesso: la sua ambientazione è in gran parte nella Svizzera dei tardi anni Trenta, con ampi squarci tuttavia anche su un contesto più esteso, quello della coeva Europa sconquassata dai totalitarismi (dai regimi nazista, fascista, ma anche da quello stalinista). La narrazione integra infatti anche una molteplicità di associazioni e di storie che si diramano senza seguire una trama principale, tanto da divergere ampiamente dalle primarie vicende di Klee e Walacek, e che contribuiscono a delineare lo scenario storico del romanzo mettendone in luce le atrocità e le sofferenze. Non è sfuggito ai critici più

successiva edizione (Orelli 20II: I5). Qualora si pianificasse una ristampa del Walacek, il refuso dovrebbe venir emendato dai suoi curatori o, in alternativa, sarebbe necessaria in questo luogo una nota al testo. 
accorti come ne Il sogno di Walceck Orelli svolga in effetti una riflessione su un particolare momento della storia svizzera ed europea - in un'intervista l'autore definirà il I 938 «uno degli anni più orribili nella storia dell'umanità» (Orelli 20Irb: 7) - e come egli integri nel romanzo anche espliciti elementi di critica a pagine della storia elvetica ${ }^{4}$. Il punto che ora preme approfondire è tuttavia un altro e riguarda, nello specifico, l'architettura discorsiva del Walacek. Il proliferare di associazioni e storie, spesso secondarie, si fonda infatti su una particolare strategia diegetica che coinvolge insistiti e puntuali riferimenti proprio al quadro contemplato da Orelli al Kunstmuseum di Berna nel 1989: Alphabet I.

\section{Variazioni orelliane su un quadro di Paul Klee}

Fino a oggi gli interpreti del Walacek, anche quelli orientati all'analisi specifica del suo rapporto con le arti figurative (cfr. Spaccini 2008), non hanno tematizzato l'importante ruolo dell'intermedialità nel romanzo, omettendo quindi di impiegare questo centrale concetto nell'analisi del dialogo sistematico che la prosa orelliana instaura con l'opera pittorica di Klee. Tra le diverse tipologie di possibili relazioni tra diversi media che sono state codificate da Irina Rajewsky, in uno studio che si è imposto come un classico in questo ambito di ricerca, è il riferimento intermediale (Rajewski 2002: I6-I8) quella che deve essere considerata per l'analisi del romanzo di Orelli. Sotto questa tipologia rientrano infatti i casi in cui un prodotto mediale descrive, evoca o tematizza un prodotto di altro medium. Tra gli esempi più tipici di questo tipo di intermedialità vi è la descrizione letteraria di opere d'arte, l'ékphrasis, un procedimento codificato come noto già nella tradizione letteraria antica. I riferimenti intermediali messi in atto da Orelli nel Walacek sono senz'altro più complessi rispetto a quest'ultima soluzione. Nel romanzo orelliano non compare infatti solo una descrizione del quadro (quindi una tradizionale ékphrasis), che pure è delineata nelle prime pagine del Walacek, ma anche ulteriori riferimenti ad Alphabet I che costellano l'intera opera e che non si limitano alle sue parti descrittive. In particolare è il richiamo a un dettaglio del quadro di Klee, la «O», a divenire una sorta

\footnotetext{
4 Nel rilevare come il romanzo di Orelli delinei «a critical panorama of Switzerland», Daniel Rothenbühler non manca di rammentare come Il sogno di Walacek esca in un anno, il I99I, in cui hanno avuto luogo le celebrazioni dei 700 anni della Confederazione elvetica. Dai festeggiamenti si sono, come noto, dissociati molti intellettuali e scrittori svizzeri in séguito alla rivelazione pubblica di come la polizia federale avesse spiato molti di essi - ma anche semplici cittadini - nel corso della guerra fredda. Orelli con la pubblicazione del Walacek - osserva Rothenbühler - «was able to circumvent both boycott and celebration, revealing the contradictions of a Switzerland as cosmopolitan as it was parochial, as magnanimous as it was petty, founded as much on freedom as on constraint» (Rothenbühler 20I2: VIII-IX).
} 
di Leitmotiv nel testo da cui scaturiscono catene di associazioni e storie. Il narratore e alcuni dei personaggi che animano l'universo narrativo del romanzo si riferiscono e si interrogano infatti su questa enigmatica «O» che solleciterà diverse associazioni e ipotesi. Lo stesso Orelli, in un'intervista apparsa dopo l'uscita del libro, offre alcuni importanti spunti per l'interpretazione di questo motivo:

\begin{abstract}
Questa $\mathrm{O}$ è diventata una specie di lente d'ingrandimento dalla quale sono scaturite un mucchio d'associazioni mie che potrebbero andare avanti all'infinito. [...] Avrei potuto intitolare il libro Blow up come il film di Antonioni: puntiamo la lente su un punto di un'immagine, apparentemente anodina, e dietro al punto scopriamo tutto un mondo che si amplia all'infinito (Orelli I99Ib: I3).
\end{abstract}

In fondo è proprio questo il principio costruttivo del romanzo: puntare il focus su un punto (un dettaglio del quadro di Klee) e da qui dar avvio a una molteplicità di associazioni e storie che si diramano senza seguire un plot unitario e concluso. Anche dall'esame dei materiali di elaborazione de Il sogno di Walacek emerge la centralità della riflessione che nel lavoro compositivo Orelli ha svolto sulla «O» di Klee. Si trovano carte (cfr. fig. 3) che presentano un vero e proprio disegno della lettera «O» ed elenchi di possibili associazioni o abbozzi di interpretazioni. Un lavoro quindi più che mai intenso su questo particolare dell'opera di Klee che ne dimostra la centralità.

A creare queste catene associative e a dare spunto a narrazioni secondarie, minori, contribuiscono i dialoghi di un gruppo di avventori di un'osteria che discutono animatamente su questo dettaglio del quadro di Klee. Nel complesso è assai ampio lo spettro associativo che viene delineato, dando spazio anche a spunti ludici nel susseguirsi delle diverse interpretazioni. Tra le possibili ipotesi, la «O» è chiamata tra l'altro a evocare: «un cerchio, ma non perfetto», «un vecchio anello ammaccato» (Orelli I99Ia: 2I); «una cornice deformata» (2I), «un o lungo e roco che sale dagli stadi» (5I), l'anello dei Nibelunghi (94), un buco nero, gouffre, abisso (63), «l'orlo di una pentola» di Nadeza Mandel'stam (57); l'ovale destinato a Sindelar, alla sua lapide (54). In questi ultimi due casi a partire dall'ipotesi associativa trae sviluppo il racconto di storie secondarie, come quella della moglie del poeta Osip Mandel'stam, costretta all'esilio dalle purghe staliniane, la quale ha conservato inizialmente le poesie del marito in una pentola, per poi impararle a memoria, per paura che gliela sequestrassero. O la storia di Matthias Sindelar, l'attaccante della nazionale austriaca - il Wunderteam - che dopo l'Anschluss dell'Austria alla Germania si rifiutò di giocare nella nazionale tedesca di Hitler e morì suicida nel 1939. Sono questi soltanto due esempi di narrazione delle numerose storie minori avviate proprio a partire dalla 


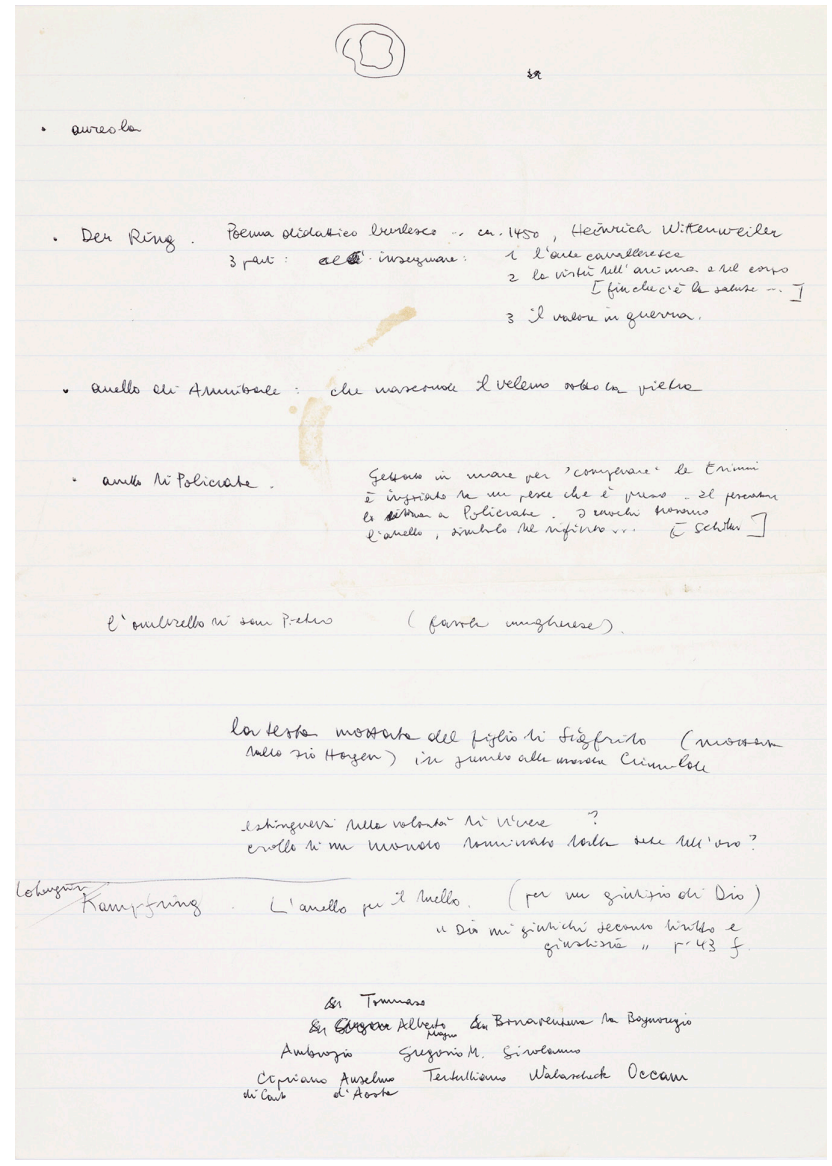

Fig. 3: Giovanni Orelli, Appunti intorno alla «O» di Klee (ASL, A-I-i/2-2).

dinamica associativa suggerita dalla «O» di Klee. Un procedimento che si fonda, quindi, su riferimenti intermediali e che costituisce una delle peculiarità più proprie della prosa del Walacek. 


\section{Bibliografia}

Bülow von, Ulrich, «Nachlässe», in M. Lepper - U. Raulff (Hg.), Handbuch Archiv. Geschichte, Aufgaben, Perspektiven, Stuttgart, Metzler, 20I6, pp. I43I52.

Orelli, Giovanni, «Mit Pro Helvetias Hilfe», Reformatio, 38, 5/6, I989, pp. 39I394.

-. Il sogno di Walaceck, Torino, Einaudi, I99Ia.

—. «Fuori dal tempo per sondare il mistero dell'uomo», intervista a cura di F. Balli, Azione, I4 novembre i99ib, p. I3.

—. «Le mie carte all'ASL», Quarto. Zeitschrift des Schweizerischen Literaturarchivs, I, I993, pp. 40-4I.

—. «Perché andare a Berna...», Quarto. Zeitschrift des Schweizerischen Literaturarchivs, 33/34, 2011a, pp. 82-89.

- «Contro la monotonia del negativo. Conversazione di riscaldamento con Giovanni Orelli», a cura di G. Boraso [aprile 20IIb], http://www.oblique. it/images/interviste/orelli_intervista_Ioaprı.pdf (consultato il I7 marzo 20I9).

-. Il sogno di Walacek, Roma, 66thand2nd, 2oIIc.

Ganzoni, Annetta, «Walaceks Traum - Giovanni Orellis erfinderische Kulturmeisterschaft», Quarto. Zeitschrift des Schweizerischen Literaturarchivs, 37, 20I3, pp. 88-94.

Rajewsky, Irina O., Intermedialität, Tübingen, Francke Verlag, 2002.

Rothenbühler, Daniel, «Introduction. A Dream-Dance on the Brink», in Giovanni Orelli, Walaschek's Dream, trad. di J. Richards, Champaign, Dalkey Archive Press, 20I2, pp. VII-XIX.

Pedroni, M. Matteo, «L'ordine e la fuga. Per Giovanni Orelli, critico militante», Il Cantonetto. Rassegna letteraria semestrale, 65, 2, 20I8, pp. I42-I54.

Puddu, Francesca, «La solidità del buio. L'evoluzione della poetica di Giovanni Orelli negli avantesti de L'anno della valanga», Versants, 6o/2, 2013a, pp. 29-42.

-. «Sirene fisse e sirene mobili. Sulla genesi di Una sirena in Parlamento di Giovanni Orelli», Strumenti critici, I32, 2013b, pp. 289-3I2.

Soldini, Fabio, «Retroscena editoriali de L'anno della valanga di Giovanni Orelli», Il Cantonetto. Rassegna letteraria semestrale, 65, 2, 20I8, pp. 52-72.

Spaccini, Jacqueline, «Quadri sognati e pittori che sognano: Il sogno di Walacek di Giovanni Orelli», in Ead., Sotto la protezione di Artemide Diana. L'elemento pittorico nella narrativa italiana contemporanea (I975-2000), Soveria Mannelli, Rubbettino, 2008, pp. I98-208. 


\section{Indice dei manoscritti}

Bern

Biblioteca nazionale svizzera

Archivio svizzero di letteratura (ASL), Archivio di Giovanni Orelli:

A-I-i Il sogno di Walacek, I99I:

A-I-i/2-I Appunti relativi ad «Alphabet I».

A-I-i/2-2 Appunti intorno alla «O» di Klee. 